\title{
Inguinofemoral lymphadenectomy and femoral dissection: cadaveric educational video
}

\author{
(D) Ilker Selcuk ${ }^{1}$, (D) Hilal Aktaş Akdemir², (D) Burak Ersak1, (D) Ilkan Tatar², (D) Mustafa Fevzi Sargon³, (D) Tayfun Güngör4 \\ 1Department of Gynecologic Oncology, Ankara City Hospital, Women's Health Education and Research Hospital, Ankara, Turkey \\ 2Hacettepe University Faculty of Medicine, Department of Anatomy, Ankara, Turkey \\ 3Lokman Hekim University Faculty of Medicine, Department of Anatomy, Ankara, Turkey \\ 4Private Clinic, Ankara, Turkey
}

\section{Abstract}

Vulvar cancer is rare. The complex inguinofemoral anatomy and the limited number of surgical procedures per year per gynecological oncologist tends to decrease the competency level. This step-by-step, cadaveric educational video was produced to increase understanding of the anatomy and technique of inguinofemoral lymphadenectomy.

Keywords: Inguinofemoral, lymphadenectomy, vulvar cancer, dissection, groin

Received: 04 February, 2019 Accepted: 26 March, 2019

\section{Introduction}

Since vulvar cancer is a rarely seen gynecological malignancy, every year only a limited number of surgeries are likely to be performed by each gynecological oncologist. Van der Zee et al. (1) suggested that at least 5 to 10 patients should be managed per year by each surgeon to ensure the competency level. Additionally, adoption of a sentinel lymph node approach has led to a less radical surgical concept that further reduces the number of cases with full inguinofemoral lymphadenectomy. The current situation has motivated gynecological oncology fellows and young surgeons to gain education and practice by cadaveric dissections. This cadaveric surgical educational video demonstrates the technique of inguinofemoral lymph node dissection in a step-by-step process.

\section{Material and Methods}

This cadaveric educational dissection video was recorded at Hacettepe University Faculty of Medicine, Department of Anatomy. One limitation of this study was that a fresh frozen cadaver was not used. However, the video was recorded to demonstrate a step-by-step technique and we believe one of the strong points of this study is that, whether the cadaver is fresh frozen or not, if used properly this type of study still has an important place in medical and post-graduate education.

\section{Inguinal anatomy}

The groin is the anatomical region between the anterolateral abdominal wall and lower extremity. The inguinal ligament, which is one edge of the femoral (Scarpa's) triangle, lies between the anterior superior iliac spine and pubic tubercle. The inguinal ligament is formed by the aponeurosis of the external oblique muscle. Under the skin the superficial fascia may be observed and it is composed of a fatty superficial layer (Camper's fascia) and a deeper membranous layer (Scarpa's fascia). Superficial vessels, which supply the lateral, middle and medial sites are the superficial circumflex iliac, superficial epigastric and superficial external pudendal vessels, respectively. Scarpa's fascia fuses with the fascia lata, the deep fascia of the thigh. Under the fascia lata the femoral vessels and nerve can be dissected. They lie as nerve-arteryvein-lymphatics formation from lateral to medial. The femoral 
vessels are covered by the femoral sheath, which is the continuation of transversalis and iliac fascia, below the inguinal ligament. Additionally, the femoral triangle is detected under the fascia lata. The lateral border is the sartorius muscle and the medial border is the adductor longus muscle. The fossa ovalis is approximately $3 \mathrm{~cm}$ inferolateral to the pubic tubercle and it is an opening through the fascia lata where the great saphenous vein enters to the femoral vein. This part is covered by a thin, multi-perforated fascia called the fascia cribrosa, and many lymphatics and venous structures pass through this region (Figure 1) $(2,3)$.

\section{Inguinal lymph nodes}

The inguinal lymph nodes are categorized into the superficial and the deep group, separated by the fascia lata. The superficial lymph nodes are located under Scarpa's fascia and divided into five groups, depending on the termination point of the great saphenous vein; superomedial, superolateral, inferomedial, inferolateral and central (4). The deep inguinal lymph nodes are located beneath the fascia lata, medial to the femoral vein (5). The lymph node found at the femoral canal, anterosuperior to the femoral vein, is known as Cloquet's node, the nexus between the deep inguinal and iliac/obturator lymph nodes (6).

\section{Surgical procedure}

This surgical procedure is performed in the low lithotomy position, at the left groin. Between the anterior superior iliac spine and pubic tubercle the skin incision is performed, $8 \mathrm{~cm}$ in length, and $2 \mathrm{~cm}$ below and parallel to the inguinal ligament. After the incision, the dissection deepens to identify Camper's fascia lying over Scarpa's fascia. Identification and preservation of Camper's fascia is critical in live patients to secure the skin flap and prevent skin necrosis. To dissect the fibrofatty tissue containing the superficial inguinal lymph nodes between the Scarpa's fascia and the fascia lata, the first step should be to mobilize the fibrofatty tissue under Scarpa's fascia and then dissect $2 \mathrm{~cm}$ cephalad, to the inguinal ligament where the aponeurosis of the external oblique muscle is seen. Afterwards, excision of the lymphatic and fibrofatty tissue is performed from lateral (superficial circumflex iliac vein) to medial (superficial external pudendal vein) and superior to inferior, identified by the inferomedial end of inguinal ligament where it intersects with the adductor longus muscle.

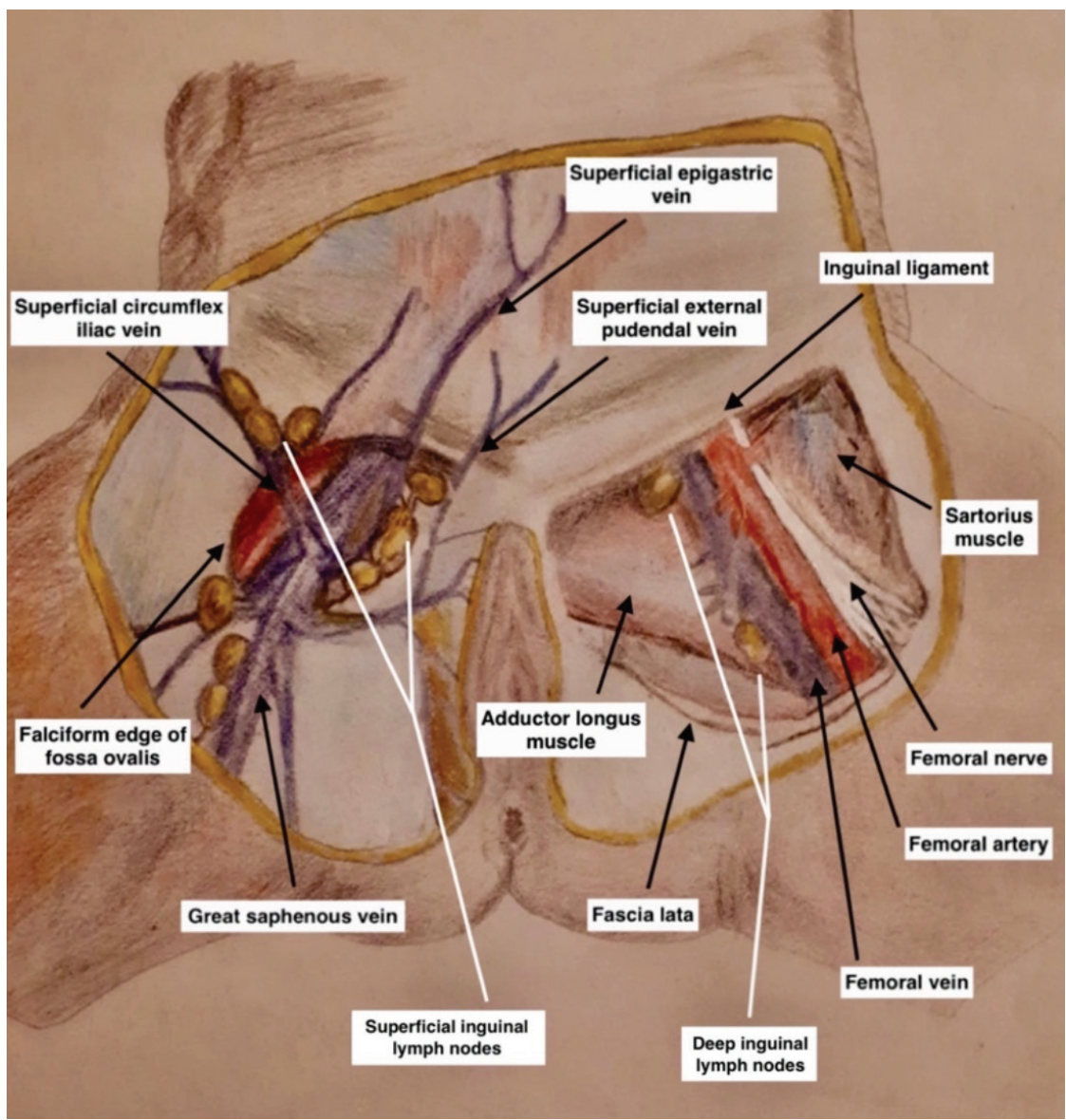

Figure 1. Inguinal anatomy with regard to superficial and deep inguinal lymph nodes (the figure was illustrated from the book "Atlas of Human Anatomy, Pelvis and Perineum, Plate 389, 7th Edition, 2019 Elsevier, Netter Frank H." by Sedef Yasin 
The fossa ovalis is encountered after resection of superficial inguinal lymph nodes (Figure 2). Medial to the falciform edge of the fossa ovalis, dissection of the cribriform fascia will lead to the deep inguinal lymph nodes, which are located medial to the femoral vein. The great saphenous vein enters the femoral vein from the opening of the fossa ovalis, so careful dissection is essential while removing the cribriform fascia. Lymphadenectomy is performed towards the apex of the femoral triangle, and there is no need to dissect the femoral sheath to excise the deep inguinal nodes completely (Figure 3). Finally, Cloquet's lymph node, the most superior of the deep inguinal nodes, may be resected from the femoral canal under the level of the inguinal ligament (7).

\section{Complications}

Hematoma, seroma, wound breakdown, wound infection and lymphedema are the most probable complications

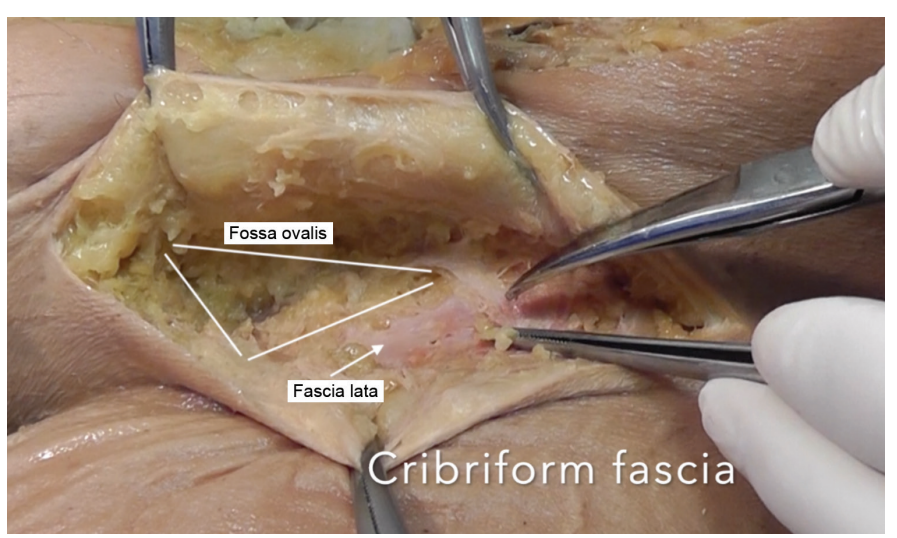

Figure 2. Cribriform fascia over the fossa ovalis, and fascia lata, after superficial inguinal lymphadenectomy

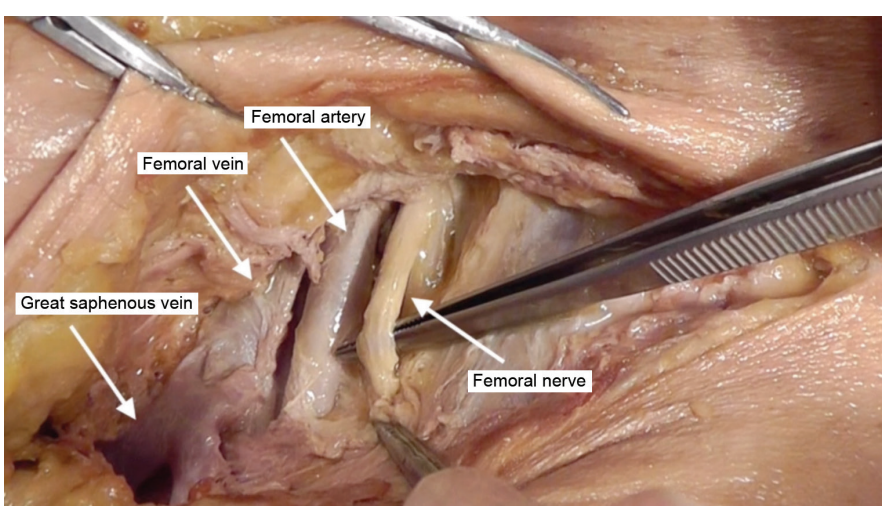

Figure 3. Femoral nerve, artery, vein and great saphenous vein after dissection of the femoral sheath after inguinofemoral lymphadenectomy. The most serious complication is lymphedema and the great saphenous vein should be secured during dissection to decrease the risk of lymphedema (8).

\section{Video 1.

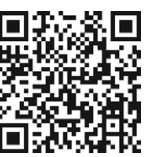

https://www.doi.org/10.4274/jtgga.galenos.2019.2019.0026.video1

Acknowledgement: Special thanks to medical illustrator Sedef Yasin. We would like to thank Hacettepe University Faculty of Medicine, Department of Anatomy, where the dissection was performed.

Conflict of Interest: No conflict of interest was declared by the authors.

Financial Disclosure: The authors declared that this study received no financial support.

\section{References}

1. Van der Zee AG, Oonk MH, De Hullu JA, Ansink AC, Vergote I, Verheijen RH, et al. Sentinel node dissection is safe in the treatment of early-stage vulvar cancer. J Clin Oncol 2008; 26: 884-9.

2. Detton AJ. Grant's Dissector. 16th ed. Philadelphia, United States: Lippincott Williams and Wilkins; 2016.

3. Netter F. Pelvis and Perineum. In: H. NF, editor. Atlas of Human Anatomy. 7th Edition. Philadelphia: Elsevier; 2019. p. Plate 389.

4. Rouvière H. Anatomy of the Human Lymphatic System. : Ann Arbor, MI: Edwards Brothers Inc; 1938.

5. Cesmebasi A, Baker A, Du Plessis M, Matusz P, Shane Tubbs R, Loukas M. The surgical anatomy of the inguinal lymphatics. Am Surg 2015; 81: 365-9.

6. Selman TJ, Luesley DM, Acheson N, Khan KS, Mann CH. A systematic review of the accuracy of diagnostic tests for inguinal lymph node status in vulvar cancer. Gynecol Oncol. 2005; 99: 20614.

7. Bristow RE. Inguinal lymphadenectomy. In: Cundiff GW, Azziz R, Bristow RE editors. Te Linde's Atlas of Gynecologic Surgery. 1st ed: Lippincott Williams \& Wilkins; 2014. p. 125-33.

8. Zhang SH, Sood AK, Sorosky JI, Anderson B, Buller RE. Preservation of the saphenous vein during inguinal lymphadenectomy decreases morbidity in patients with carcinoma of the vulva. Cancer 2000; 89: $1520-5$. 